\title{
Effect of Bias Electrode Position on Terahertz Radiation from Pentagonal Mesas of Superconducting $\mathrm{Bi}_{2} \mathrm{Sr}_{2} \mathrm{CaCu}_{2} \mathrm{O}_{8+\delta}$
}

\author{
Kaveh Delfanazari $^{1,2 *}$, Hidehiro Asai ${ }^{3}$, Manabu Tsujimoto ${ }^{2}$, Takanari Kashiwagi ${ }^{2}$, Takeo \\ Kitamura $^{2}$, Takashi Yamamoto ${ }^{4}$, Wade Wilson ${ }^{5}$, Richard A. Klemm ${ }^{5}$, Toshiaki Hattori ${ }^{2}$, and \\ Kazuo Kadowaki ${ }^{2}$ \\ ${ }^{1}$ Optoelectronics Research Centre and Centre for Photonic Metamaterials, University of \\ Southampton, Highfield, Southampton, SO17 1BJ, UK \\ ${ }^{2}$ Graduate School of Pure and Applied Sciences, University of Tsukuba, 1-1-1, Tennodai, Tsukuba, \\ Ibaraki, 305-8573, Japan \\ ${ }^{3}$ Electronics and Photonics Research Institute, The National Institute of Advanced Industrial \\ Science and Technology (AIST), Tsukuba, Ibaraki 305-8568, Japan \\ ${ }^{4}$ Wide Bandgap Materials Group, National Institute for Materials Science, 1-1 Namiki, Tsukuba, \\ Ibaraki 305-0044, Japan \\ ${ }^{5}$ Department of Physics, University of Central Florida, Orlando, FL 32816, USA \\ *kd2n12@orc.soton.ac.uk
}

\begin{abstract}
We examined the effect of the bias current position on the radiation of intense and coherent terahertz electromagnetic waves from the intrinsic Josephson junctions (IJJs) in regular pentagonal mesas of single crystalline high$T_{c}$ superconducting $\mathrm{Bi}_{2} \mathrm{Sr}_{2} \mathrm{CaCu}_{2} \mathrm{O}_{8}+\delta$. The emission from Sample 1, a mesa with a current bias feed on its middle, is strong and sharply peaked at $0.47 \mathrm{THz}$, whereas that from Sample 2, a mesa with its feed along an edge, shows a comparably strong, sharp peak at $0.43 \mathrm{THz}$. The common backbending in the current-voltage characteristics due to Joule heating was seen for Sample 1 but almost not for Sample 2. The experimental results are in good agreement with numerical simulations and calculations, both for the cavity resonances and the angular emission patterns. The resonance peaks obtained from these pentagonal mesas are both stronger and narrower than those found previously from differently shaped mesas fabricated by focused ion beam milling. The overall devices are small enough to be cooled to their operational temperatures by Stirling refrigerators, so that properly designed devices could be held in one hand as for laser pointers. Therefore, our IJJ-based
\end{abstract}

emitter devices are promising candidates to fill the $\mathrm{THz}$ gap with compact, continuouswave quantum solid-state sources.

KEYWORDS: Intrinsic Josephson junctions, Superconductor, Terahertz wave, Cavity resonance, Ac-Josephson effect, Terahertz emitters.

\section{INTRODUCTION}

The observation of coherent terahertz $(\mathrm{THz})$ electromagnetic (EM) waves from a mesa structure of the high transition temperature $T_{\mathrm{c}}$ cuprate superconductor $\mathrm{Bi}_{2} \mathrm{Sr}_{2} \mathrm{CaCu}_{2} \mathrm{O}_{8+\delta}(\mathrm{Bi}-$ 2212) was first reported in 2007 [1]. Single crystalline Bi-2212 behaves as a stack of intrinsic Josephson junctions (IJJs), and has a large superconducting energy gap (60 meV) [1-3]; Bi-2212 can generate EM waves with frequencies in the $\mathrm{THz}$ range by the application of a $d c$-voltage $V$ across the $N$ active IJJs, each $1.533 \mathrm{~nm}$ thick, stacked along the $c$-axis of the mesa, with emission frequency $f$ satisfying the quantum $a c$ Josephson relation $f=f_{\mathrm{J}}=(2 e / h) V / N=$ $483.597891 \mathrm{GHz}$ for $V / N=1 \mathrm{mV}$, where $e$ is the electric charge and $h$ is Planck's constant [4-5]. Although IJJ mesas are small in size, $\sim 100 \mathrm{~mm}$ across and $\sim 1 \mathrm{~mm}$ thick, they can 
generate high power $\mathrm{THz}$ waves up to $\sim 30 \mu \mathrm{W}$ [6-10], and the power generated by a threemesa array was reported to be $610 \mu \mathrm{W}$ [11]. Thus, the IJJ-based $\mathrm{THz}$ emitter is one of the promising candidates to fill the $\mathrm{THz}$ gap with a compact, continuous-wave, quantum solid state source [5-33]. Its small size allows it to be cooled to its operational temperature range of $40-60 \mathrm{~K}$ by a Stirling refrigerator, so it can be held in one hand for many applications.

Although the uniform part of the $a c$-Josephson current is the primary radiation source, its nonuniform part can excite one or more EM cavity resonances, which can amplify the radiation at the allowed EM cavity resonance frequencies $f_{\mathrm{c}}(n m)$, where $n, m$ are integers indexing the transverse magnetic (TM) modes. These primary and secondary mechanisms have been mostly identified from studies of rectangular and disk mesas [6-18]. The radiation can be enhanced by locking the $a c$ Josephson frequency $f_{\mathrm{J}}$ onto a particular cavity resonance frequency $f_{\mathrm{c}}(\mathrm{nm})$ appropriate for the mesa geometry and dimensions [16].

In this paper we discuss our recent experimental and numerical studies of the $\mathrm{THz}$ emission from regular pentagonal mesas, a sketch of which is shown in Fig. 1. Pentagonal cavities are interesting to investigate because of their high power capability, relative to other geometries e.g. rectangular and elliptic, as waveguides and transmission lines [34-36]. They also produce circular polarization with only one probe feed for many telecommunication application purposes. Here we find that they also show stronger resonances with narrower bandwidths in comparison to previously studied cavities, e.g. triangular mesas [28-30].

One mesa has its bias feed on its center (Sample 1 with $\mathrm{T}_{\mathrm{c}}=68.6 \mathrm{~K}$, Fig. 2, left), and the second has its bias feed along an edge (Sample 2 with $\mathrm{T}_{\mathrm{c}}=67.5 \mathrm{~K}$, Fig. 2, right). Our measurements of the current-voltage $(I-V)$ characteristics (IVCs) and of the resulting emission spectra obtained from the Fouriertransform infrared (FT-IR) spectrometer are presented. Sub-THz radiation was observed over the frequency range of $0.41 \mathrm{THz}$ to 0.53 $\mathrm{THz}$ for bath temperatures $T$ ranging from 30
$\mathrm{K}$ to $55 \mathrm{~K}$. Although this frequency spectrum is broadly tunable, it exhibits a sharp peak at $0.47 \mathrm{THz}$ for Sample 1 and at $0.43 \mathrm{THz}$ for Sample 2. Since analytic calculations of the $\mathrm{TM}$ modes of regular pentagonal mesas are not available, we numerically studied them using a finite difference time domain (FDTD) method. The numerical calculation resulted in a single cavity mode frequency that is in good agreement with the sharp peak frequency observed in the experiment on Sample 1. The angular distribution of the emission at this frequency was measured, and fit to the dual source model of the uniform $a c$ Josephson current source and of the excitation of the fundamental cavity resonance mode of a diskshaped antenna.

\section{SAMPle PREPARATION METHODS}

A Bi-2212 single crystal grown by the traveling solvent floating zone (TSFZ) technique was used in this study [37]. To control the doping level in order to obtain slightly under-doped crystals important for $\mathrm{THz}$ radiation, a piece of the crystal with lateral sizes of $10 \times 5 \mathrm{~mm}^{2}$ was annealed at $650{ }^{\circ} \mathrm{C}$ for 24 hours in argon gas mixed with $0.1 \%$ oxygen. By using silver paste, a piece of $800 \times 800 \mu \mathrm{m}^{2}$ in size of this crystal was glued onto a sapphire substrate. Immediately after cleaving the crystal with Scotch tape, thin layers of silver and gold were evaporated onto its freshly cleaved surface.

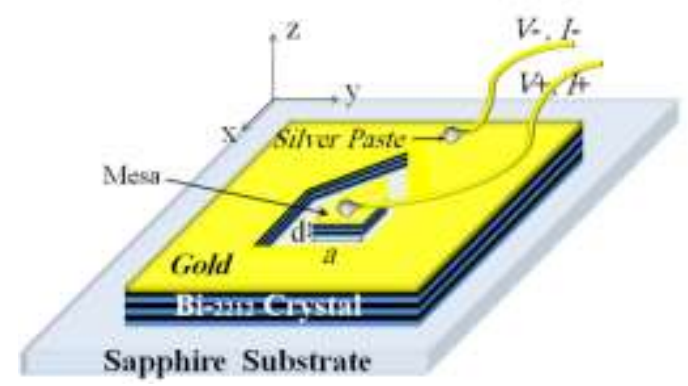

Fig. 1 (Colour online) Schematic view of the pentagonal mesa, Sample 1, with $\boldsymbol{T}_{\mathbf{c}}=\mathbf{6 8 . 6} \mathrm{K}$. The crystal surface contains thin layers of $\mathrm{Ag}$ and $\mathrm{Au}$. The current is applied along the $c$-axis direction of the $\mathrm{Bi}-2212$ crystal using gold wires.

Regular pentagonal mesas were then fabricated by FIB milling, and leads were 
attached as sketched for Sample 1 in Fig. 1. For Sample 2, the lead was attached along an edge. A scanning ion microscopy (SIM) image of Sample 1 (left) and an optical microscope image of Sample 2 (right) are shown in Fig. 2. By using an atomic force microscope (AFM), the dimensions of the mesa were measured, resulting in a clear trapezoidal cross-sectional shape (not shown here).

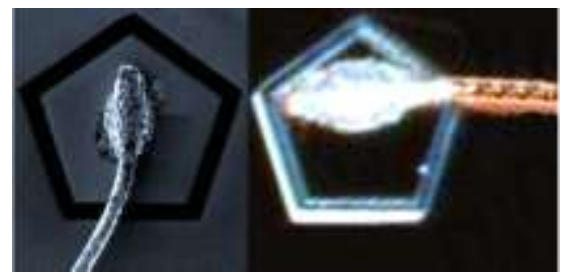

Fig. 2 (Colour online) A scanning ion microscopy image of Sample 1 with current bias feed on the middle (left) and an optical microscope image of Sample 2 with current bias feed along an edge (right).

Finally, in order to apply the $d c$-current $I$ to the mesas [26], gold wires $10 \mu \mathrm{m}$ in diameter were attached to the surfaces as electrodes using silver paste. A function generator with a load resistor of 0-300 $\Omega$ was used in order to bias each mesa. Two different commercial digital multimeters were used to monitor $I$ and $V$ applied to the mesas. The $\mathrm{THz}$ radiation was detected by the liquid helium-cooled Sibolometer. The FT-IR spectrometer (JASCO FARIS-1) was used to measure the emission frequency.

\section{EXPERIMENTAL RESULTS}

Here we present the experimental results obtained from two nearly identical regular pentagonal mesas with same top edge length $a$ $=60 \mu \mathrm{m}$. A typical example of the IVCs and the radiation intensity detected by the $\mathrm{Si}$ bolometer is shown simultaneously in Fig. 3 at $T=40.0 \mathrm{~K}$ for Sample 1. In this figure, the left vertical and horizontal scales indicate the applied $I$ and $V$, respectively, whereas the right vertical scale indicates the intensity of the $\mathrm{THz}$ emission detected by the Si-bolometer. As seen in Fig. 3, we observed $\mathrm{THz}$ radiation on the return branch region where the current is relatively low, $I=2 \sim 3 \mathrm{~mA}$, and $V=0.55 \sim 0.8$ $\mathrm{V}$. A clear irreversible (IR) type $\mathrm{THz}$ radiation $[12,26]$ at bath temperature $T=40.0 \mathrm{~K}$ for this sample was seen. As $I$ is increased from 0, Fig. 3 shows that no radiation occurs until $I$ reaches $10 \mathrm{~mA}$, at which the mesa jumps to the resistive state at $V=1.2 \mathrm{~V}$.
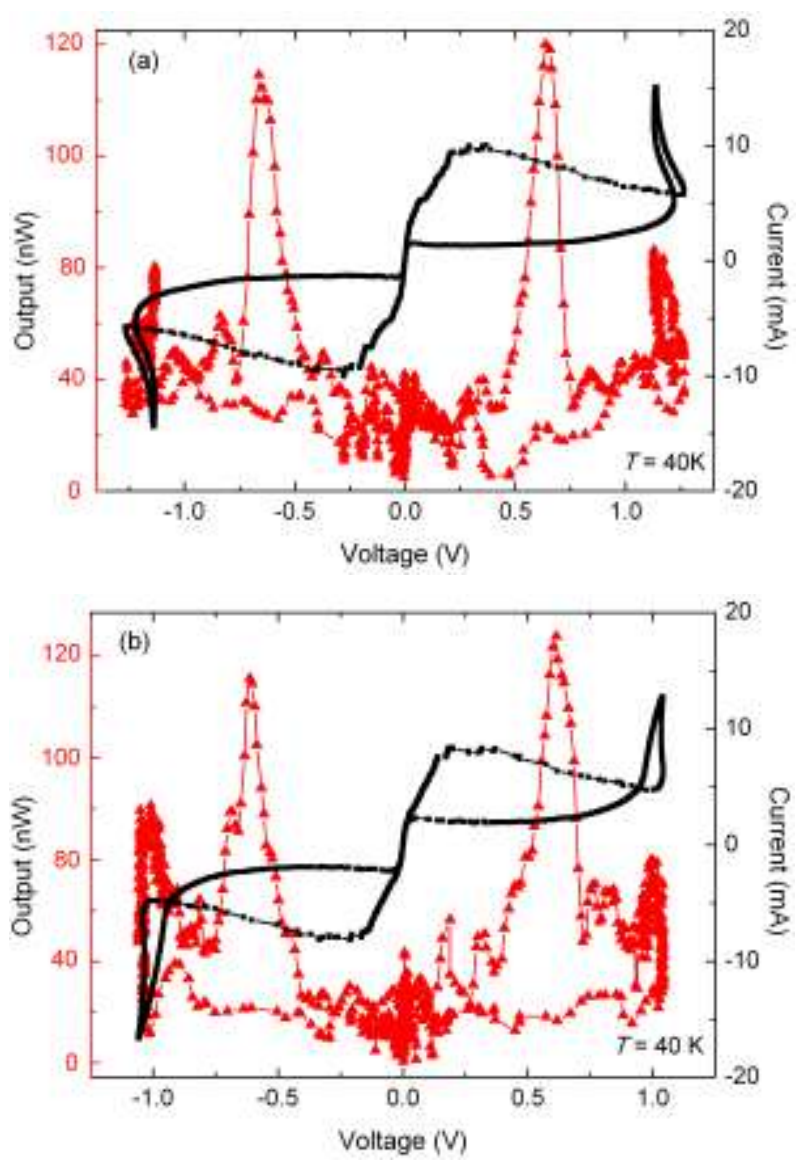

Fig. 3 (Colour online) Experimental results for pentagonal mesas: (black) typical IVCs at the bath temperature $T=40.0 \mathrm{~K}$ and (red) the emission intensity detected by the Si-bolometer. (a) Sample 1, (b) Sample 2 .

Along with the rapid jump in the voltage, a small hysteresis in the outermost branch of the $I-V$ curve for currents between about 8 $\mathrm{mA}$ and $12 \mathrm{~mA}$ appears. The negative differential resistance due to Joule heating $[12,16]$ is also seen in the IVCs of Sample 1 for voltage range $1.1<\mathrm{V}<1.25$.

Figure 3 (b) shows $\mathrm{THz}$ radiation from Sample 2. As displayed in the IVCs, the $I_{\mathrm{c}}$ is around $8 \mathrm{~mA}$ and $\mathrm{THz}$ radiation was observed for $I=2 \sim 3 \mathrm{~mA}$ and $V=0.45 \sim 0.85$ $\mathrm{V}$. The maximum emission bias voltage for 
Sample 2 is $\sim 0.9 \mathrm{~V}$, lower than that of Sample 1. As a result, the negative resistance and backbending behavior that appeared above $1 \mathrm{~V}$ in the IVCs of Sample 1 are almost absent. These results suggest that when the bias feed is along an edge, the mesa doesn't over heat as much, resulting in higher output emission power. Figure 3 (b) shows that emission power peak is both increased and broadened. We attribute this to the effect of the bias feed position on the $\mathrm{THz}$ radiation from pentagonal mesas. From the measurement of the output voltage in our Si-bolometer, and from the angular emission distribution (see Fig. 7 (b) and also the text in section V), we estimate the integrated output power from sample 1 as $\sim 0.5 \mu \mathrm{W}$ [6]. We note that these pentagonal mesas were fabricated by FIB milling of a Bi-2212 crystal (the simplest and fastest procedure that also offers the high level of data reproducibility), so their substrates are part of the same crystal. As we reported previously $[6,8,16,17]$, replacing the $\mathrm{Bi}-2212$ substrate with a conducting one, producing a so-called stand-alone mesa, can greatly enhance the emission power.

For both samples, we observed $\mathrm{THz}$ radiation with $T$ ranging from $30 \mathrm{~K}$ to $55 \mathrm{~K}$. In Fig. 4, we show the frequency spectrum of the overall emission observed in those $T$ ranges for both samples. As seen in this figure, the emission power is widely tunable over the frequency range of $0.41 \mathrm{THz}$ to $0.53 \mathrm{THz}$, but has a sharp peak at $0.47 \mathrm{THz}$ for Sample 1 (Fig. 4a) and at $0.43 \mathrm{THz}$ for Sample 2 (Fig. 4b). In order to understand the physical origin of the strong emission at these frequencies, we numerically investigated the cavity resonance modes of a regular pentagonal mesa that has its current bias feed in the middle.
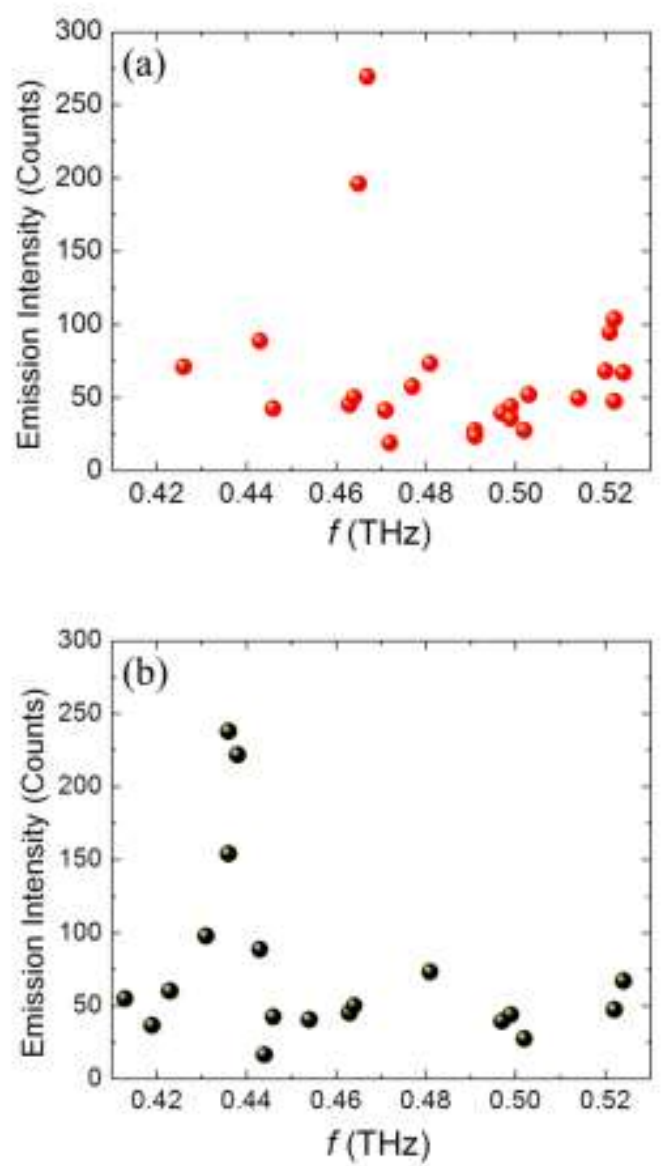

Fig. 4 (Colour online) The overall emission power for $30 \mathrm{~K} \leq \mathrm{T} \leq 55 \mathrm{~K}$ as a function of frequency for (a) Sample 1 (red circles) and (b) Sample 2 (black circles).

\section{NUMERICAL RESULTS}

In this section, the results of our FDTD calculations of the cavity resonance modes in a regular pentagonal resonator are presented. In this investigation, the EM fields inside and outside of the IJJs are simultaneously calculated by assuming that the motion of the phase differences across each pair of IJJ layers inside the mesa are perfectly synchronized [38]. Figure 5 shows a sketch of the numerical calculation model. Here, the region where the current lead is attached to the mesa is considered to be a "hot spot". This hot spot (thermal inhomogeneity) is presumed to be induced by local heating at the contact region between the current lead and the mesa. By studying the hot spots with a laser scanning technique, previous workers claimed that the appearance of the hot spot is important for the synchronization of the radiation from the different layers $[14,15,18,19]$. 


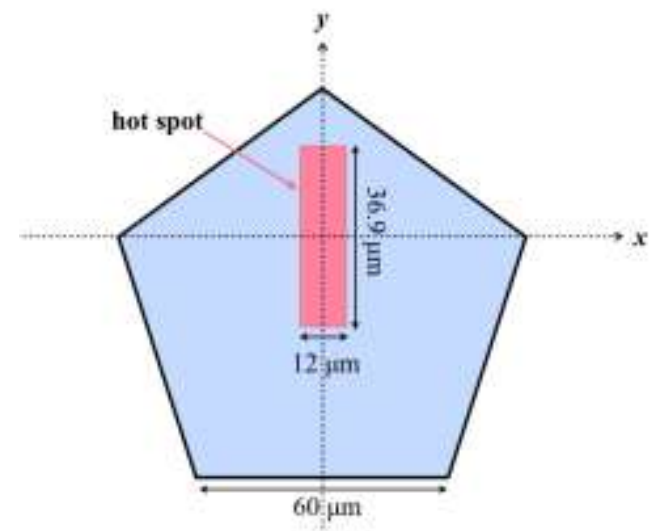

Fig. 5 (Colour online) Sketch of the numerical calculation model, showing the hot current injection spot and the sample dimensions.

Theoretical studies also predicted that the excitation of a cavity resonance mode is important for the synchronization $[38,39]$. However, by using the photoluminescence from attached $\mathrm{SiC}$ microcrystals, it was shown that high power emission can occur without a hot spot, and it was claimed that the excitation of an electromagnetic cavity mode was the most important mechanism for achieving high power and synchronized emission [40,41].

Bias current injection extended over the length of a rectangular mesa was also found to lead to increased power [42], as did control of the hot spot by a laser [43]. But, as previously suggested [16,17], stand-alone mesas constructed with the rectangular mesa covered both top and bottom by $\mathrm{Au}$ and sandwiched between insulating outer layers of either $\mathrm{MgO}$ or sapphire were found to be effective in removing the heating, and allowing for emission frequencies well above $1 \mathrm{THz}[21,44]$.

We modeled the heating effects by the creation of an inhomogeneous critical current. The critical current of the region shown by the redcolored rectangle in Fig. 5 is assumed to be 75 $\%$ of that of the rest of the sample. The parameters used in the numerical calculation are as follows: the $c$-axis conductivity is taken to be $2 \mathrm{~S}, \lambda_{c}$, the $c$-axis penetration depth, is assumed to be $100 \mu \mathrm{m}$, the dielectric constant within each junction is 17.64, and the thicknesses of the superconducting and insulating layers are $0.3 \mathrm{~nm}$ and $1.2 \mathrm{~nm}$, respectively. These values are comparable to those of Bi-2212 samples [38]. The emission power spectrum obtained from our numerical calculation is shown in Fig. 6 (a). In this figure, a sharp resonance-like peak appears around $0.47 \mathrm{THz}$. This frequency is almost equal to the peak frequency observed in the experiment on Sample 1. In Fig. 6 (b), we show a snap shot of the electric field amplitude, $E_{\mathrm{z}}$, at this frequency. A standing wave mode that is symmetric across the $y$-axis is clearly apparent. Note that the signs of the amplitude $E_{z}$ at the top and bottom of the mesa are different.
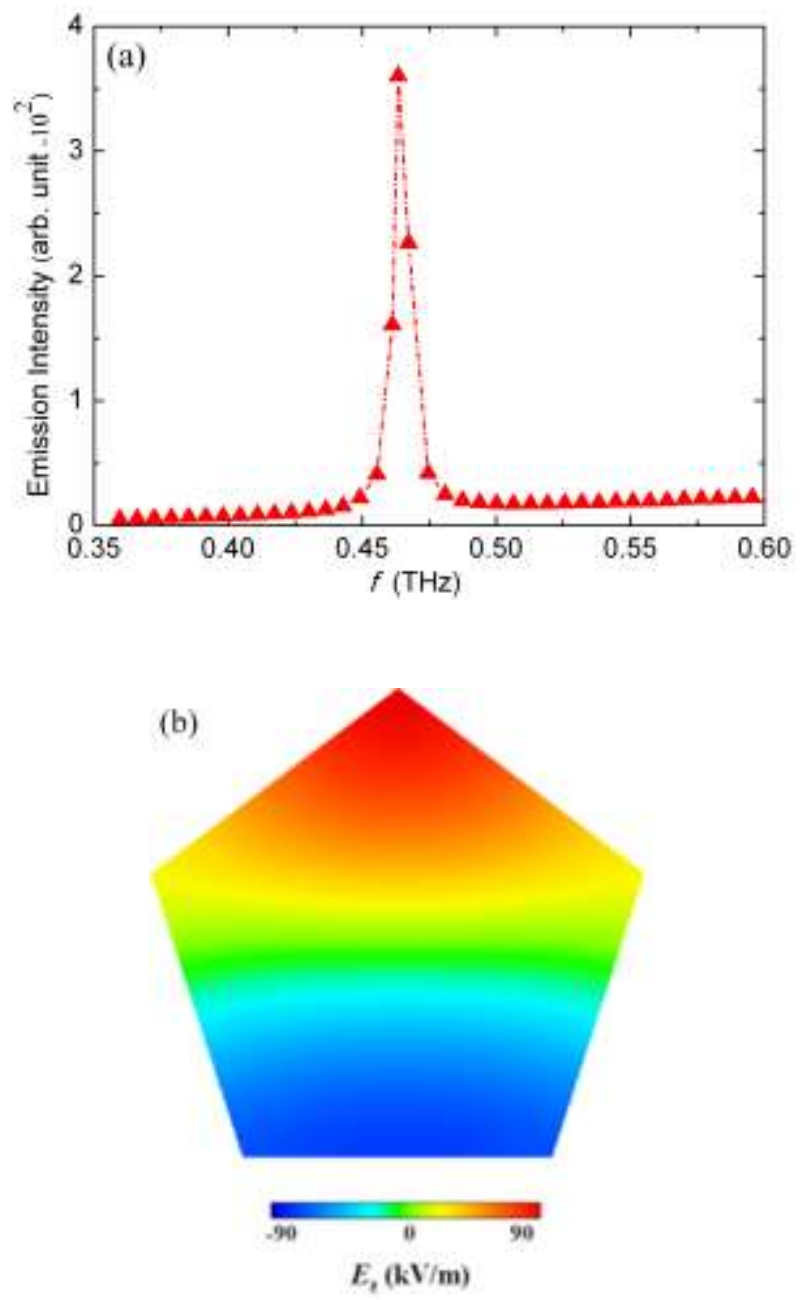

Fig. 6 (Color online) (a) The emission power spectrum obtained from the numerical calculation. (b) The map of the electric field amplitude, $E_{\mathrm{z}}$, at $0.47 \mathrm{THz}$.

\section{ANGULAR DISTRIBUTION STUDIES}

We also measured the angular distribution of the emission from Sample 1 at the large 
peak at $0.47 \mathrm{THz}$, and our results are shown in Fig. 7 (b).

Although no analytic solutions of the TM modes in a thin regular pentagonal antenna have been published, a number of studies have been made [43-46].
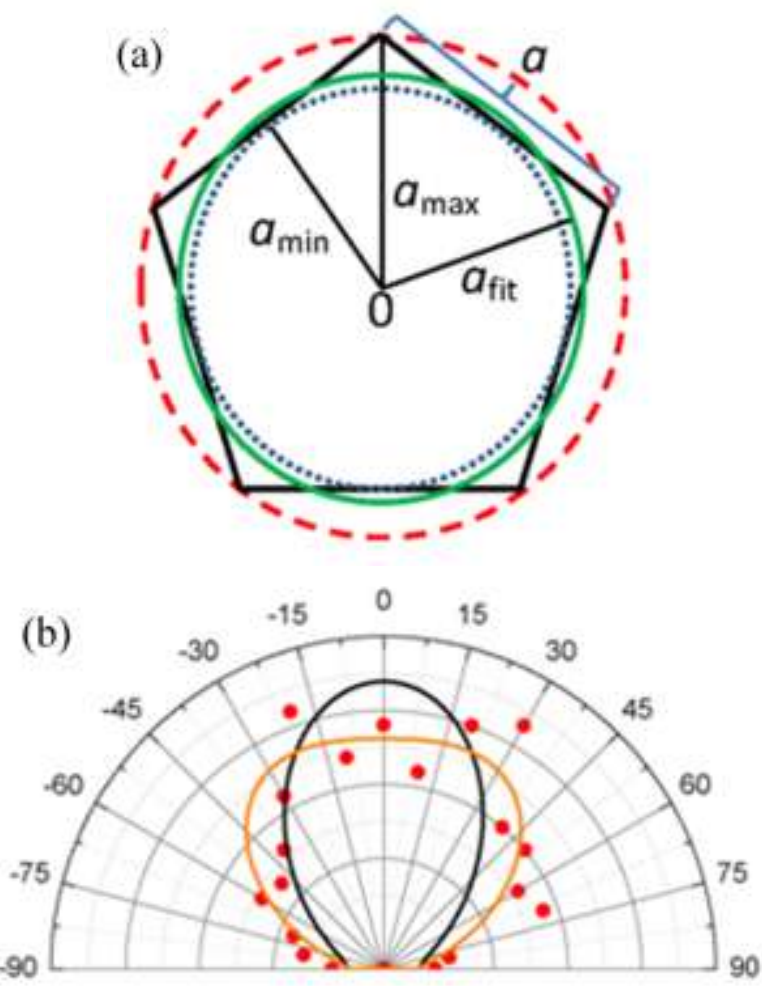

Fig. 7 (Color online) (a) Sketch of a pentagonal mesa of side $a$ with inner (dotted blue) and outer (dashed red) circles of radii $a_{\min }$ and $a_{\max }$. (b) Angular distribution of the radiation at $0.47 \mathrm{THz}$ from Sample 1 (red dots), along with the best (11) circular cavity fit (black) and the overall best dual-source fit (orange). Horizontal axis shows $I(\theta) / I(0)$. See text.

The simplest picture was constructed by Ravelo and Mazari and by Zheng et al. $[46,48]$. Following their suggestions, we modeled the pentagonal mesa of side $a$ as a circular- or disk-shaped mesa, as sketched in Fig. 7 (a), with the minimum radius $a_{\min }=(a / 2) \tan (3 \pi / 10) \approx 0.688 a$, and the maximum radius $a_{\max }=a /[2 \sin (\pi / 5)] \approx 0.851 a$. In the fits, we assumed the dual-source model that was successfully used to fit the emission from disk mesas [12,16,17]. The peak emission frequency at $0.47 \mathrm{THz}$ for Sample 1 is assumed to be at the fundamental cavity mode frequency $f_{11}$ of a disk of radius $a_{\text {fit }}$, given by $f_{11}=c \chi_{11} /\left(2 \pi a_{\mathrm{fit}} n_{\mathrm{r}}\right)$, where $\mathrm{n}_{\mathrm{r}} \approx 4.2$ is the index of refraction and $\chi_{11}=1.8412$. Taking $f_{11}=0.47 \mathrm{THz}$, we obtain $a_{\mathrm{fit}}=44.9$ $\mu \mathrm{m}$, or $0.749 a$.

Hence, this fit corresponds to the solid green circle in Fig. 7(a), so that $a_{\min }<a_{\mathrm{fit}}<a_{\mathrm{max}}$, as required for a good fit. For Sample 2 the peak at $0.43 \mathrm{THz}$ corresponds to $a_{\mathrm{fit}}=48.3 \mu \mathrm{m}$, or $0.806 a$, also satisfying $a_{\min }<a_{\mathrm{fit}}<a_{\max }$, but with $a_{\text {fit }}$ much closer to $a_{\max }$ than for Sample 1 . This difference is most likely due to the different placement of the current leads in the two samples. We fit the angular distribution from Sample 1 using two models, the cavity source model and the dual-source model used previously $[12,16,17]$. The dual source and cavity source fits are shown by the orange and black curves in Fig. 7 (b). The standard deviations for the fits to the Sample 1 emission angular distribution data for the cavity source model and the dual-source model were 0.385 and 0.241 , respectively. The best fit is therefore the dual source fit, which corresponds to about $50 \%$ of each source contributing to the radiation power at 0.47 THz. This is comparable to values obtained for disk mesas [12].

In our studies of the emission from equilateral triangular mesas, the fundamental cavity mode is 6-fold degenerate, with even and odd modes about the bisectors of each of the three vertex angles [49]. At the fundamental frequency for the two modes defined by one vertex, the emission patterns predicted are essentially identical to that for the fundamental disk cavity mode, shown as Fig. 4(a) in [16], except that the even and odd mode patterns lie in perpendicular planes. Hence, with the additional 3-fold vertex degeneracy, such patterns rotated by multiples of $30^{\circ}$ about the $z$-axis should be possible. Extending such models to regular pentagons would lead to the suggestion of a set of five such patterns rotated successively by $72^{\circ}$ about the $z$-axis, plus another set of 5 identical patterns in planes perpendicular to theirs. The combined pattern would be indistinguishable from that of a disk with an arbitrary wave function nodal line direction [16]. To extract more information 
would require at least two orders of magnitude more experimental data points. More important, one would also need angular studies of the emission at higher frequencies, where the emission patterns for equilateral triangles are very different from those of a disk [49]. Such calculations are currently in progress for regular pentagonal antennas [50], and we suggest that future studies of sandwich stand-alone regular pentagonal mesas be made at much higher emission frequencies. We expect that those studies at frequencies well above 1 $\mathrm{THz}$ could lead to observable cavity emission enhancement features not present in disk mesas.

Hence, we conclude that modeling the pentagonal mesa as a disk for the purposes of studying the angular distribution of the radiation is justified, especially since exact solutions of the cavity modes are not available.

\section{Conclusions}

By studying the $I-V$ characteristics and the emission spectra experimentally and performing the numerical FDTD calculations, the $\mathrm{THz}$ radiation from pentagonal IJJs mesa structures of superconducting Bi-2212 was investigated in this paper. The radiation was found to be broadly tunable from 0.41 to 0.53 $\mathrm{THz}$ in low bias region. The mesa with a current bias feed on its middle shows a sharp peak at $0.47 \mathrm{THz}$, whereas mesa with its feed along an edge shows a sharp peak at a lower frequency $(0.43 \mathrm{THz})$ and intensity. The tunability from the outer current-voltage characteristic branch is not as broad as that observed from triangular and disk mesas fabricated using focused ion beam milling, but the intensity is higher. The peak frequency observed in the experiment is in good agreement with the cavity resonance frequency estimated by the numerical calculations using the FDTD method. The angular distribution of the radiation at the peak frequency was measured, and was well fit by modeling the pentagonal mesa as a disk, using the dualsource model of the uniform ac Josephson current source and the fundamental disk cavity resonance frequency. Further studies of standalone mesa sandwich structures of regular pentagonal mesas could lead to very interesting and practical results. Arrays constructed from such structures could provide the best devices to fill the $\mathrm{THz}$ gap.

\section{REFERENCES}

[1] L. Ozyuzer, A. E. Koshelev, C. Kurter, N. Gopalsami, Q. Li, M. Tachiki, K. Kadowaki, T. Yamamoto, H. Minami, H. Yamaguchi, T. Tachiki, K. E. Gray, W.-K. Kwok, and U. Welp, Science 318 (2007) 1291-1293.

[2] K. Kadowaki, H. Yamaguchi, K. Kawamata, T. Yamamoto, H. Minami, I. Kakeya, U. Welp, L. Ozyuzer, A. Koshelev, C. Kurter, K. E. Gray, and W.-K. Kwok, Physica C 468 (2008) 634-639.

[3] R. Kleiner, F. Steynmeyer, G. Kunkel, and P. Müller, Phys. Rev. Lett. 68 (1994) 2394.

[4] B. D. Josephson, Phys. Lett. 1 (1962) 251-253.

[5] U. Welp, K. Kadowaki, and R. Kleiner, Nat. Photon. 7 (2013) 702.

[6] S. Sekimoto, C. Watanabe, H. Minami, T. Yamamoto, T. Kashiwagi, R. A. Klemm, K. Kadowaki, Appl. Phys. Lett. 103 (2013) 182601.

[7] K. Kadowaki, M. Tsujimoto, K. Delfanazari, T. Kitamura, M. Sawamura, H. Asai, T. Yamamoto, K. Ishida, C. Watanabe, S. Sekimoto, K,. Nakade, T. Yasui, K. Asanuma, T. Kashiwagi, H. Minami, M. Tachiki, T. Hattori, and R. A. Klemm, Physica C 491 (2013) 2.

[8] T. Kitamura, T. Kashiwagi, T. Yamamoto, M. Tsujimoto, C. Watanabe, K. Ishida, S. Sekimoto, K. Asanuma, T. Yasui, K. Nakade, Y. Shibano, Y. Saiwai, H. Minami, R. A. Klemm, and K. Kadowaki, Appl. Phys. Lett. 105 (2014) 202603.

[9] D. Y. An, J. Yuan, N. Kinev, M. Y. Li, Y. Huang, M. Ji, H. Zhang, Z. L. Sun, L. Kang, B. B. Jin, J. Chen, J. Li, B. Gross, A. Ishii, K. Hirata, T. Hatano, V. P. Koshelets, D. Koelle, R. Kleiner, H. B. Wang, W. W. Xu, and P. H. Wu, Appl. Phys. Lett. 102 (2013) 092601.

[10] F. Turkoglu, H. Koseoglu, Y. Demirhan, L. Ozyuzer, S. Preu, S. Malzer, Y. Simsek, P. Müller, 
T. Yamamoto, and K. Kadowaki, Supercond.

Sci. Technol. 25 (2012) 125004.

[11] T. M. Benseman, K. E. Gray, A. E. Koshelev, W.-K. Kwok, U. Welp, H. Minami, K. Kadowaki, and T. Yamamoto, Appl. Phys. Lett. 103 (2013) 022602

[12] M. Tsujimoto, K. Yamaki, K. Deguchi, T. Yamamoto, T. Kashiwagi, H. Minami, M. Tachiki, K. Kadowaki, and R. A. Klemm, Phys. Rev. Lett. 105 (2010) 037005.

[13] T. Kashiwagi, K. Yamaki, M. Tsujimoto, K. Deguchi, N. Orita, T. Koike, R. Nakayama, H. Minami, T. Yamamoto, R.A. Klemm, M Tachiki, and K. Kadowaki, J. Phys. Soc. Jpn. 80 (2011) 094709 .

[14] H. B. Wang, S. Guénon, J. Yuan, A. Iishi, S. Arisawa, T. Hatano, T. Yamashita, D. Koelle, and R. Kleiner, Phys. Rev. Lett. 102 (2009) 017006.

[15] H. B. Wang, S. Guénon, B. Gross, J. Yuan, Z. G. Jiang, Y. Y. Zhong, M. Grünzweig, A. Iishi, P. H. Wu, T. Hatano, D. Koelle, and R. Kleiner, Phys. Rev. Lett. 105 (2010) 057002.

[16] R. A. Klemm and K. Kadowaki, J. Phys.: Condens. Matter 22 (2010) 375701.

[17] R. A. Klemm and K. Kadowaki, J.

Supercond. Novel Magn. 23 (2010) 613.

[18] S. Guénon, M. Grünzweig, B. Gross, J. Yuan, Z. G. Jiang, Y. Y. Zhong, M. Y. Li, A. Iishi, P. H. Wu, T. Hatano, R. G. Mints, E. Goldobin, D. Koelle, H. B. Wang, and R. Kleiner, Phys. Rev. B 82 (2010) 214506.

[19] B. Gross, S. Guénon, J. Yuan, M. Y. Li, J. Li, A. Ishii, R. G. Mints, T. Hatano, P. H. Wu, D. Koelle, H. B. Wang, and R. Kleiner, Phys. Rev. B 86 (2012) 094524

[20] M. Li, J. Yuan, N. Kinev, J. Li, B. Gross, S. Guénon, A. Ishii, K. Hirata, T. Hatano, D. Koelle, R. Kleiner, V. P. Koshelets, H. Wang, and P. Wu, Phys. Rev. B 86 (2012) 060505.

[21] M. Ji, J. Yuan, B. Gross, F. Rudau, D. Y. An, M. Y. Li, X. J. Zhou, Y. Huang, H. C. Sun, Q. Zhu, J. Li, N. Kinev, T. Hatano, V. P. Koshelets, D. Koelle, R. Kleiner, W. W. Xu, B. B. Jin, H. B. Wang, and P. H. Wu, Appl. Phys. Lett. 105 (2014) 122602 .
[22] X. Hu and S. Lin, Phys. Rev. B 80 (2009) 064519 .

[23] S. Lin and X. Hu, Phys. Rev. Lett.100 (2008) 247006.

[24] A. E. Koshelev, Phys. Rev. B 78 (2008) 174509.

[25] S. Savel'ev, V. A. Yampol'skii, A. L. Rakhmanov and F. Nori, Rev. Prog. Phys. 73 (2010) 026501 .

[26] T. Kashiwagi, M. Tsujimoto, T. Yamamoto, H. Minami, K. Yamaki, K. Delfanazari, K. Deguchi, N. Orita, T. Koike, R. Nakayama, T. Kitamura, M. Sawamura, S. Hagino, K. Ishida, K. Ivanovic, H. Asai, M. Tachiki, R. A. Klemm, and K. Kadowaki, Jpn. J. Appl. Phys. 51 (2012) 010113 .

[27] M. Tsujimoto, T. Yamamoto, K. Delfanazari, R. Nakayama, T. Kitamura, M. Sawamura, T. Kashiwagi, H. Minami, M. Tachiki, K. Kadowaki, and R. A. Klemm, Phys. Rev. Lett. 108 (2012) 107006.

[28] K. Delfanazari, H. Asai, M. Tsujimoto, T. Kashiwagi, T. Kitamura, T. Yamamoto, M. Sawamura, K. Ishida, M. Tachiki, R. A. Klemm, T. Hattori, and K. Kadowaki, Physica C 491 (2013) 16.

[29] K. Delfanazari, H. Asai, M. Tsujimoto, T. Kashiwagi, T. Kitamura, T. Yamamoto, M. Sawamura, K. Ishida, C, Watanabe, S. Sekimoto, H. Minami, M. Tachiki, R. A. Klemm, T. Hattori, and K. Kadowaki, Optics Express, 21 (2013) 2171-2184.

[30] R. A. Klemm, K. Delfanazari, M. Tsujimoto, T. Kashiwagi, T. Kitamura, T. Yamamoto, M. Sawamura, K. Ishida, T. Hattori, and K. Kadowaki, Physica C 491 (2013) 30.

[31] T.M. Benseman, A. E. Koshelev, K. E. Gray, W.-K. Kwok, U. Welp, K. Kadowaki, M. Tachiki, T. Yamamoto, Phys. Rev. B 84 (2011) 064523.

[32] Yu. M. Shukrinov, I. R. Rahmonov, A. Plecenik, P. Seidel, E. Il'ichev and W. Nawrocki, Supercond. Sci. Technol. 27 (2014) 124007.

[33] V. M. Krasnov, Phys. Rev. B 83 (2011) 174517. 
[34] C.S. Kenney and P.L. Overfelt, IEEE

Trans. Electromag. Compa.34 (1992) 3.

[35] R. S. Rhen, Q. Zhihua, K. W. Leung and D. Wang, Microwave Opt. Technol. Lett. 27 125. (2000)

[36] R. S. Chen, M. Lei, K. F. Tsang, and D. Wang, Microwave Opt. Technol. Lett. 31

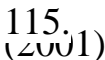

[37] T. Mochiku, and K. Kadowaki, Physica C 235 (1994) 523.

[38] H. Asai, M. Tachiki, and K. Kadowaki, Phys. Rev. B 85 (2012) 064521.

[39] A. E. Koshelev and L. N. Bulaevskii, Phys. Rev. B 77 (2008) 014530.

[40] H. Minami, C. Watanabe, K. Sota, S. Sekimoto, T. Yamamoto, T. Kashiwagi, R. A. Klemm, and K. Kadowaki, Phys. Rev. B 89 (2014) 054503 .

[41] C. Watanabe, H. Minami, T. Yamamoto, T. Kashiwagi, R. A. Klemm, and K. Kadowaki, J. Phys.: Condens. Matter 26 (2014) 172201.

[42] M. Tsujimoto, H. Kambara, Y. Maeda, Y. Yoshioka, Y. Nakagawa, and I. Kakeya, Phys. Rev. Appl. 2 (2014) 044016.

[43] C. Watanabe, H, Minami, T. Kitamura, K. Asanuma, K. Nakade, T. Yasui, T. Yamamoto, T. Kashiwagi, R. A. Klemm, and K. Kadowaki, Appl. Phys. Lett. 106 (2015) 042603.

[44] T. Kashiwagi, T. Yamamoto, T. Kitamura, K. Asanuma, C. Watanabe, K. Nakade, T. Yasui, Y. Saiwai, Y. Shibano, H. Minami, M. Tsukimoto, R. Yoshizaki, K. Delfanazari, R. A. Klemm, and K. Kadowaki, (unpublished)

[45] J.-L. Cao, W.-L. Dong, and W.-X. Li, IEEE Trans. Ant. Prop. AP-34 (1986) 103.

[46] B. Ravelo and B. Mazari, Prog.

Electromag. Res. M 12 (2010) 95.

[47] L. Brás, N. Borges Carvallo, and P. Pinho, IEEE Trans. Ant. Prop. 60 (2012) 1634.

[48] Q. Zheng, J. Peng, B. Yao, R. Zhong, W. $\mathrm{Xu}, \mathrm{T}$. Xiang, and $\mathrm{L}$. $\mathrm{Li}, \quad$ Second Int. Conf. on
Mechanic Automation and Control Engineering (MACE), (2011) 1076.

[49] R. A. Klemm, C. Reid, W. Wilson, M. Morales, D. Cerkoney, K. Delfanazari, and K. Kadowaki (unpublished).

[50] M. Morales, K. Delfanazari, K. Kadowaki, and R. A. Klemm (unpublishelished). 